\section{Altitude Measurement of Low Elevation Target Based on Iterative Subspace Projection}

GUANGHUI ZHAO

GUANGMING SHI

Xidian University

\begin{abstract}
A new temporal-spatial general MUSIC (TS-GMUSIC) method is proposed for the altitude measurement of low-elevation targets. As an expansion of the traditional temporal-spatial process, our proposed method employs the alternate projection (AP) theory to measure the target elevation. Different from conventional AP where the direction vector is iteratively projected to achieve the elevation, we iteratively project the received echoes onto different signal subspace to obtain the elevation. Thus the conventional direction-search of the multiple targets can be simplified into one target parameter estimation. Further we consider the multipath geometry characteristic of low-elevation targets, therefore the direction-search of both direct wave and specular wave is changed into one-dimensional parameter search. The Monte-Carlo simulations prove that the proposed TS-GMUSIC shows better performance than the existing temporal-spatial algorithms. Moreover, the real-data processing of VHF radar demonstrates the validity of the proposed method.
\end{abstract}

Manuscript received January 26, 2008; revised August 17, 2009; released for publication January 6, 2010.

IEEE Log No. T-AES/47/2/940843.

Refereeing of this contribution was handled by C. Baker.

This work was supported by the National Science Foundation of China under Grants 60736043, 60776795, 60902079, and 60902031, by the Basic Science Research Fund of Xidian University K50510020018, and by the Specialized Research Fund for the Doctoral Program of Higher Education under Grant 200807010004.

Authors' address: Key Laboratory of Intelligent Perception and Image Understanding, Ministry of Education, Xidian University, Xi'an, Shannxi 710071, China, E-mail: (ghzhao@mail.xidian.edu.cn).

$\overline{0018-9251 / 11} / \$ 26.00$ (c) 2011 IEEE

\section{INTRODUCTION}

Radar tracking of low-elevation targets [1-4] has been a challenging problem since the emergence of missile technology. During the tracking process, due to the reflections from sea or ground, serious degradation will occur in the altitude estimation of a target located within one elevation beamwidth of the radar antenna above the horizon. Considering the phase-controlled array radar observing two independent targets in the same range cell, if the two targets are not resolvable in the range or Doppler dimensions, a beam oriented in the direction of one target will pick up the energy from the other one. Therefore it is very difficult to make accurate measurements of either target.

In the past several decades, many digital beamforming (DBF) techniques have been presented, such as Capon's minimum variance (MV) method [5] and maximum likelihood (ML) estimation [6]. However, since the resolution of the beam is strictly limited by the aperture of the array, the tracking beamwidth can not be made small enough to separate targets. From the point of improving the angle resolution, the concept of virtual array [7-10] was proposed, in which array mapping $[7,8]$ and high-order cumulant $[9,10]$ are two popular techniques, but the computational load of these two techniques is very high.

Recently, considerable effort has been spent in developing high-resolution techniques [11-15] for estimating the direction-of-arrival (DOA) of multiple signals. The MUSIC algorithm [11] and ESPRIT algorithm [12] are based on the subspace decomposition, so that they can provide the excellent performance in high signal-to-noise ratio (SNR), long data records, and spatially uncorrelated sources field. However, when the signal is perfectly correlated (coherent), especially in multipath propagation, the subspace decomposition based algorithms suffer from the loss of rank of the covariance matrix, yielding erroneous results. Usually this problem is solved via the spatial smoothing approach [13, 14], but spatial smoothing requires a large array of antennas to compensate the aperture loss. The alternate projection (AP) approach [15] proposed by Ilan Ziskin and Mati Wax can separate the coherent targets without aperture loss, since this method estimates the target position by making use of a deterministic multipath propagation model parameterized on the target elevation or altitude. However, the computational load increases stupendously with the increase of the target number.

To achieve more precise angle estimation, the concept of the temporal-spatial cascaded process was proposed in [16], where the temporal-spatial ML (TS-ML) algorithm is used to estimate the DOA of the multiple Doppler signals by employing both the temporal and spatial structures of these signals. It has been shown that the TS-ML algorithm outperforms 
the ML algorithm which only uses the spatial structure of signals, especially in the case of less snapshots and low SNR [4]. But the TS-ML algorithm is more expensive in computational cost since it enlarges the size of the dimensions to be searched. This limits its applications in practical systems.

In this paper, a new low-elevation temporal-spatial general MUSIC (TS-GMUSIC) approach is proposed, in which the idea of the AP is also utilized. In the proposed method, after the pulse compression, echoes containing different target information in the same range cell are collected together. Then by projecting the target echoes into the constructed signal subspace, one of the low-elevation targets can be distinguished from the mixed echoes. The general MUSIC algorithm is employed to achieve low elevation. The proposed TS-GMUSIC method can be used for DOA estimation of multiple low-elevation spatially-distributed sources in the far field. The simulation results and the real-data processing of VHF radar demonstrate the validity of the proposed method.

This paper is organized as follows. Section II presents the multipath signal model. In Section III we propose the TS-GMUSIC method for altitude measurement. In Section IV the experimental results and the real-data processing are given. The conclusion is drawn in Section V.

\section{MULTIPATH PROPAGATION MODEL}

With the rapid development of missile technology, how to distinguish and attack the low-elevation flying target becomes a challenging problem in current radar signal processing. In the low-elevation circumstance, the target is normally within a beamwidth of the horizon. The reflections from the surface of the Earth affect the estimation greatly. Fig. 1 shows the target model in this circumstance, where $R_{d}$ denotes the range of the direct wave of the target, $H_{r}$ denotes the height of the center of the $i$ th receiving array above the sea level, $H_{T}$ is the altitude of the target, $\phi_{d}$ is the angle of the direct path ray measured upward with respect to broadside to the array, while $\phi_{s}$ is the angle of the specular path ray measured downward from broadside to the array.

It can be found that the direct target echo, as well as the specular component (multipath echo), is received at the terminal with small time delay. In this paper, we only consider the multipath echo, since the effect of its reflected energy is much greater than that of the diffuse components [1]. Since the multipath component is highly correlated with the direct echo and it always lies in the beamwidth of the receiver, it is very difficult to detect the altitude of the target. Fig. 2 shows a typical application when the two targets in the same range emerge in the radar display terminal.

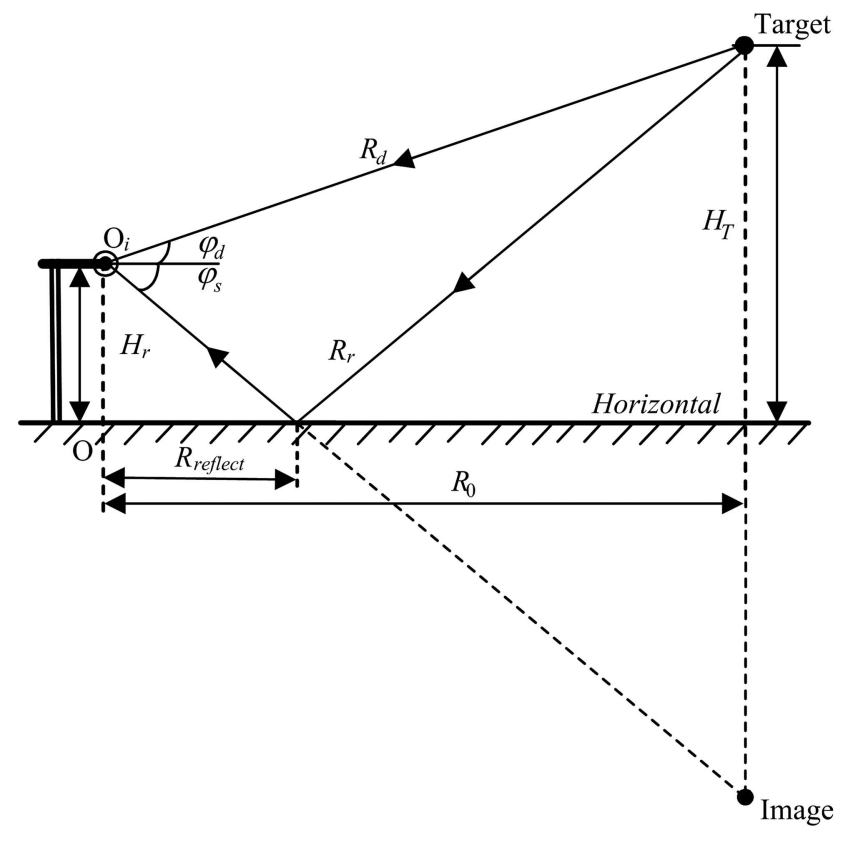

Fig. 1. Multipath model.

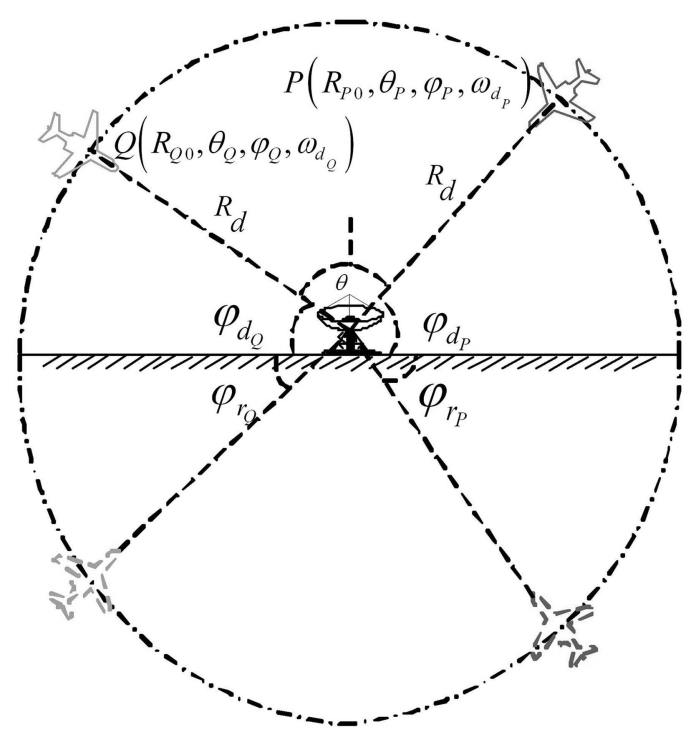

Fig. 2. Typical application model.

Here we take a uniform linear array with $N$ isotropic sensors into account, in which each sensor has the same receiving model as depicted in Fig. 1. The distance between two adjacent sensors is assumed to be a half of the wavelength corresponding to the central carrier-frequency of the signal. Suppose $P$ narrowband plane waves with known waveforms impinging on this array from distinct angles relative to the array normal. As a special case of coherent two-ray multipath, considering the symmetric multipath problem encountered in a low-elevation radar tracking environment [1], the received echo of the array sensors can be expressed as

$$
\mathbf{X}(t)=\mathbf{A}_{N \times P}(\phi) \mathbf{S}(t)+\mathbf{u}(t)
$$


where $\mathbf{X}(t)=\left[x_{1}(t), x_{2}(t), \ldots, x_{N}(t)\right]^{\mathrm{T}}, i=1,2, \ldots, N, \mathrm{~T}$ denotes the transpose, $x_{i}(t)$ is the $i$ th output vector of the array antenna; $\mathbf{A}_{N \times P}(\phi)=\left[\mathbf{a}\left(\phi_{1}\right), \mathbf{a}\left(\left(\phi_{2}\right), \ldots, \mathbf{a}\left(\phi_{P}\right)\right]\right.$ is the spatial direction matrix of the $P$ targets; $\mathbf{S}(t)=\left[s_{1}(t), s_{2}(t), \ldots, s_{P}(t)\right]^{\mathrm{T}}, s_{k}(t)=s_{k} \exp \left(j \omega_{d_{k}} t\right), s_{k}$ and $\omega_{d_{k}}$ are the signal amplitude and the Doppler frequency of the $k$ th target respectively; and $\mathbf{u}(t)=$ $\left[u_{1}(t), u_{2}(t), \ldots, u_{N}(t)\right]^{\mathrm{T}}$ is the background noise which $\quad$ The above equation can be expanded into

$$
\begin{aligned}
& X_{M}=\left[\begin{array}{c}
s_{1}(1)\left[a\left(\phi_{d_{1}}\right)-\rho a\left(\phi_{s_{1}}\right)\right]+s_{2}(1)\left[a\left(\phi_{d_{2}}\right)-\rho a\left(\phi_{s_{2}}\right)\right] \cdots+s_{P}(1)\left[a\left(\phi_{d_{P}}\right)-\rho a\left(\phi_{s_{P}}\right)\right] \\
s_{1}(2)\left[a\left(\phi_{d_{1}}\right)-\rho a\left(\phi_{s_{1}}\right)\right]+s_{2}(2)\left[a\left(\phi_{d_{2}}\right)-\rho a\left(\phi_{s_{2}}\right)\right] \cdots+s_{P}(2)\left[a\left(\phi_{d_{P}}\right)-\rho a\left(\phi_{s_{P}}\right)\right] \\
\vdots \\
s_{1}(M)\left[a\left(\phi_{d_{1}}\right)-\rho a\left(\phi_{s_{1}}\right)\right]+s_{2}(M)\left[a\left(\phi_{d_{2}}\right)-\rho a\left(\phi_{s_{2}}\right)\right] \cdots+s_{P}(M)\left[a\left(\phi_{d_{P}}\right)-\rho a\left(\phi_{s_{P}}\right)\right]
\end{array}\right]+\mathbf{U} \\
& =\left[\begin{array}{ccccccc}
e^{j \omega_{d_{1}} T_{r}} a\left(\phi_{d_{1}}\right) & e^{j \omega_{d_{1}} T_{r}} a\left(\phi_{s_{1}}\right) & e^{j \omega_{d_{2}} T_{r}} a\left(\phi_{d_{2}}\right) & e^{j \omega_{d_{2}} T_{r}} a\left(\phi_{s_{2}}\right) & \cdots & e^{j \omega_{d_{P}} T_{r}} a\left(\phi_{d_{P}}\right) & e^{j \omega_{d_{P}} T_{r}} a\left(\phi_{s_{P}}\right) \\
e^{j \omega_{d_{1}} 2 T_{r}} a\left(\phi_{d_{1}}\right) & e^{j \omega_{d_{1}} 2 T_{r}} a\left(\phi_{s_{1}}\right) & e^{j \omega_{d_{2}} 2 T_{r}} a\left(\phi_{d_{2}}\right) & e^{j \omega_{d_{2}} 2 T_{r}} a\left(\phi_{s_{2}}\right) & \cdots & e^{j \omega_{d_{P}} 2 T_{r}} a\left(\phi_{d_{P}}\right) & e^{j \omega_{d_{P}} 2 T_{r}} a\left(\phi_{s_{P}}\right) \\
\vdots & & & & & \\
e^{j \omega_{d_{1}} M T_{r}} a\left(\phi_{d_{1}}\right) & e^{j \omega_{d_{1}} M T_{r}} a\left(\phi_{s_{1}}\right) & e^{j \omega_{d_{2}} M T_{r}} a\left(\phi_{d_{2}}\right) & e^{j \omega_{d_{2}} M T_{r}} a\left(\phi_{s_{2}}\right) & \cdots & e^{j \omega_{d_{P}} M T_{r}} a\left(\phi_{d_{P}}\right) & e^{j \omega_{d_{P}} M T_{r}} a\left(\phi_{s_{P}}\right)
\end{array}\right]\left[\begin{array}{c}
s_{1} \\
-\rho s_{1} \\
s_{2} \\
-\rho s_{2} \\
\vdots \\
s_{p} \\
-\rho s_{p}
\end{array}\right] \\
& =\left[\mathbf{a}_{s t}\left(\phi_{1}, \omega_{d_{1}}\right), \mathbf{a}_{s t}\left(\phi_{2}, \omega_{d_{2}}\right), \ldots, \mathbf{a}_{s t}\left(\phi_{P}, \omega_{d_{P}}\right)\right] \cdot\left[\begin{array}{lllll}
s_{1} & -\rho s_{1} & s_{2} & -\rho s_{2} \cdots s_{p} & -\rho s_{p}
\end{array}\right]^{\mathrm{T}}+\mathbf{U} \\
& =A_{s t}\left(\phi, \omega_{d}\right) S_{2 P}+\mathbf{U}
\end{aligned}
$$

is assumed to be spatially white and uncorrelated with the $P$ signals.

Considering the circumstance of targets of low elevation and the influence of symmetric multipath, the signal subspace $\mathbf{a}\left(\phi_{k}\right)$ is spanned by linear combinations of target directions vectors of the form

$$
\mathbf{a}\left(\phi_{k}\right)=\mathbf{a}\left(\phi_{d k}\right)+r_{k} \mathbf{a}\left(\phi_{s k}\right), \quad k=1,2, \ldots, P
$$

where $\mathbf{a}\left(\phi_{d_{k}}\right)$ is the direction vector of the direct wave of the $k$ th target; $\mathbf{a}\left(\phi_{s_{k}}\right)$ is the corresponding direction vector of the multipath; $r_{k}=\rho \exp \left(j \Delta \varphi_{k}\right)$ is the surface reflection coefficient, $\rho$ is the magnitude of the surface reflection coefficient, and $\Delta \varphi_{k}=\pi$ at low grazing angle for horizontal polarization and flat ground. Through the discrete sampling, $M$ samples of the radar echo are available after the range compression or matched-filtering process, and the discrete form of the received echo in (1) can be expressed as

$$
\mathbf{X}_{M}(m)=\mathbf{A}_{N \times P}(\phi) \mathbf{S}_{M}(m)+\mathbf{u}(m), \quad m=1,2, \ldots, M
$$

where $\mathbf{S}_{M}(m)=\left[s_{1}(m), s_{2}(m), \ldots, s_{P}(m)\right]^{\mathrm{T}}, s_{i}(m)=$ $s_{i} \exp \left(j \omega_{d_{i}} m T_{r}\right)$, and $T_{r}$ is the pulse repeat interval. All the $M$ discrete samples can be summarized as where $\mathbf{a}_{s t}\left(\phi_{i}, \omega_{d_{i}}\right)=\left[\mathbf{a}_{t}\left(\omega_{d_{i}}\right) \otimes \mathbf{a}\left(\phi_{d_{i}}\right), \mathbf{a}_{t}\left(\omega_{d_{i}}\right) \otimes \mathbf{a}\left(\phi_{s_{i}}\right)\right]$ is the temporal-spatial steering vector, $\otimes$ denotes the Kronecker product, $\mathbf{a}_{t}\left(\omega_{d_{i}}\right)=\left[\exp \left(j \omega_{d_{i}} T_{r}\right), \exp \left(j \omega_{d_{i}} 2 T_{r}\right)\right.$ $\left., \ldots, \exp \left(j \omega_{d_{i}} M T_{r}\right)\right]^{\mathrm{T}}$ is the temporal steering vector; and $\mathbf{U}=[\mathbf{u}(1), \mathbf{u}(2), \ldots, \mathbf{u}(M)]^{\mathrm{T}}$ is the noise matrix.

\section{THE PROPOSED TS-GMUSIC ESTIMATION}

A. Brief Introduction of the Temporal-Spatial ML Algorithm

In the TS-ML algorithm [16], the Doppler frequency of the $k$ th target can be obtained by the following expression

$$
\begin{aligned}
& \left(\hat{\phi}, \hat{\omega}_{d}\right)=\arg \max _{\phi, \omega_{d}}\left[\mathbf{X}^{\mathrm{H}}\left(\omega_{d_{1}}\right) \mathbf{a}\left(\phi_{d_{1}}\right), \mathbf{X}^{\mathrm{H}}\left(\omega_{d_{1}}\right) \mathbf{a}\left(\phi_{s_{1}}\right), \ldots,\right. \\
& \left.\mathbf{X}^{\mathrm{H}}\left(\omega_{d_{P}}\right) \mathbf{a}_{s}\left(\phi_{d_{P}}\right), \mathbf{X}^{\mathrm{H}}\left(\omega_{d_{P}}\right) \mathbf{a}\left(\phi_{S_{P}}\right)\right] B^{-1} \\
& \times\left[\mathbf{X}^{\mathrm{H}}\left(\omega_{d_{1}}\right) \mathbf{a}\left(\phi_{d_{1}}\right), \mathbf{X}^{\mathrm{H}}\left(\omega_{d_{1}}\right) \mathbf{a}\left(\phi_{s_{1}}\right), \ldots,\right. \\
& \left.\mathbf{X}^{\mathrm{H}}\left(\omega_{d_{P}}\right) \mathbf{a}_{s}\left(\phi_{d_{P}}\right), \mathbf{X}^{\mathrm{H}}\left(\omega_{d_{P}}\right) \mathbf{a}\left(\phi_{s_{P}}\right)\right]^{\mathrm{H}}
\end{aligned}
$$

where $\mathbf{X}^{\mathrm{H}} \mathbf{a}_{s t}\left(\phi_{i}, \omega_{d_{i}}\right)=\mathbf{X}^{\mathrm{H}}\left[\mathbf{a}_{t}\left(\omega_{d_{i}}\right) \otimes \mathbf{a}\left(\phi_{d_{i}}\right), \mathbf{a}_{t}\left(\omega_{d_{i}}\right) \otimes\right.$

$\left.\mathbf{a}\left(\phi_{s_{i}}\right)\right]=\left[\mathbf{X}^{\mathrm{H}}\left(\omega_{d_{i}}\right) \mathbf{a}\left(\phi_{d_{i}}\right), \mathbf{X}^{\mathrm{H}}\left(\omega_{d_{i}}\right) \mathbf{a}\left(\phi_{s_{i}}\right)\right], i=1,2, \ldots, P$,

$\mathbf{X}^{\mathrm{H}}\left(\omega_{d_{i}}\right)=\left[x_{1}\left(\omega_{d_{i}}\right), x_{2}\left(\omega_{d_{i}}\right), \ldots, x_{N}\left(\omega_{d_{i}}\right)\right]^{\mathrm{T}}$ is the output of array after Fourier transform; $\mathbf{B}=$ $\mathbf{A}_{s t}^{\mathrm{H}} \mathbf{A}_{s t}=\left(\mathbf{A}_{s}^{\mathrm{H}} \mathbf{A}_{s}\right) \odot\left(\mathbf{A}_{t}^{\mathrm{H}} \mathbf{A}_{t}\right), \odot$ denotes the Hadamard 
product, $\mathbf{A}_{s}(\phi)=\left[\mathbf{a}_{s}\left(\phi_{1}\right), \mathbf{a}_{s}\left(\phi_{2}\right), \ldots, \mathbf{a}_{s}\left(\phi_{P}\right)\right], \mathbf{a}_{s}\left(\phi_{i}\right)$ is the steering vector from direction $\phi_{i}, \mathbf{A}_{t}\left(\omega_{d}\right)=$ $\left[\mathbf{a}_{t}\left(\omega_{d_{1}}\right), \mathbf{a}_{s}\left(\omega_{d_{2}}\right), \ldots, \mathbf{a}_{s}\left(\omega_{d_{P}}\right)\right], \mathbf{a}_{t}\left(\omega_{d_{i}}\right)$ is the steering vector of $i$ th target in the temporal domain.

We can see that (6) can be viewed as a multidimensional iterative problem. When estimating the elevation and Doppler of the target, the TS-ML uses the traditional ML-AP method [6], which adopts the alternate maximization (AM) technique. The AM technique is a conceptually simple way for multidimensional maximization. In every iteration procedure, the maximization is performed with respect to one parameter while others are fixed.

\section{B. Our Proposed Method Based on Iterative Projection}

From the analysis given above, we can find that in ML-AP algorithm, it is the direction vector that is iteratively projected to obtain different signal subspace. In this paper, the AM technique is also used. The difference is that it is the signal echo that is iteratively projected onto different signal subspace to obtain the elevation. Now we propose the idea of TS-GMUSIC.

After the process of pulse compression or matched-filtering, the targets in different ranges can be distinguished by the range cell detection. We take the targets within the same range cell into account. The initial target angles, including azimuth and elevation $\left(\hat{\theta}_{j}^{(0)}, \hat{\phi}_{d_{j}}^{(0)}\right), j=1,2, \ldots, P$, can be estimated via the DBF. Then the target subspace can be denoted as $\hat{P}_{\left(\hat{\theta}_{j}^{(0)}, \hat{\phi}_{j}^{(0)}\right.}$, which can be constructed by the reconstructed target echo $\hat{S}_{\left(\hat{\theta}_{j}^{(0)}, \hat{\phi}_{j}^{(0)}\right)}$; the detailed reconstruction process can refer to the example depicted in Fig. 1 in the section below

$$
\hat{P}_{\left(\hat{\theta}_{j}^{(0)}, \hat{\phi}_{j}^{(0)}\right)}=\hat{S}_{\left(\hat{\theta}_{j}^{(0)}, \hat{\phi}_{j}^{(0)}\right)}\left[\left(\hat{S}_{\left(\hat{\theta}_{j}^{(0)}, \hat{\phi}_{j}^{(0)}\right)}\right)^{\mathrm{H}} \hat{S}_{\left(\hat{\theta}_{j}^{(0)}, \hat{\phi}_{j}^{(0)}\right)}\right]\left(\hat{S}_{\left(\hat{\theta}_{j}^{(0)}, \hat{\phi}_{j}^{(0)}\right)}\right)^{\mathrm{H}} .
$$

In the $(k+1)$ th iteration, to obtain the parameter of the first target $\hat{x}_{1}^{(k+1)}$, we write the received echo as the mixture of multiple target echoes

$$
\begin{aligned}
\mathbf{X} & =\hat{x}_{1}^{(k+1)}+\hat{x}_{2}^{(k+1)}+\cdots+\hat{x}_{P}^{(k+1)} \\
& =\hat{x}_{1}^{(k+1)}+\hat{P}_{\left(\hat{\theta}_{2}^{(k)}, \hat{\phi}_{2}^{(k)}\right)} \mathbf{X}+\cdots+\hat{P}_{\left(\hat{\theta}_{P}^{(k)}, \hat{\phi}_{P}^{(k)}\right)} \mathbf{X} \\
& =\hat{x}_{1}^{(k+1)}+\sum_{i=2}^{P} \hat{P}_{\left(\hat{\theta}_{i}^{(k)}, \hat{\phi}_{P}^{(k)}\right)} \mathbf{X} \\
& =\hat{x}_{1}^{(k+1)}+\hat{P}_{\left(\hat{\theta}_{(2 \sim P)}^{(k)} \hat{\phi}_{(2 \sim P)}^{(k)}\right)} \mathbf{X} .
\end{aligned}
$$

Thus the direction parameters of the first target can be estimated from the received echo after projection

$$
\hat{x}_{1}^{(k+1)}=\left(\mathbf{I}-\hat{P}_{\left(\hat{\theta}_{(2 \sim P)}^{(k)} \hat{\phi}_{(2 \sim P)}^{(k)}\right)}\right) \mathbf{X} .
$$

Then using (9), the target angles of the first target $\left(\hat{\theta}_{1}^{(k+1)}, \hat{\phi}_{d_{1}}^{(k+1)}, \hat{\phi}_{s_{1}}^{(k+1)}\right)$ can be achieved. With the same process, the parameters of other targets, $\left(\hat{\theta}_{j}^{(k+1)}, \hat{\phi}_{d_{j}}^{(k+1)}, \hat{\phi}_{s_{j}}^{(k+1)}\right), j=2, \ldots, P$, can also be obtained. After the elevation $\hat{\phi}_{i}^{(k+1)}$ is obtained, the Doppler estimation of the $i$ th target in (6) is changed into

$$
\begin{aligned}
\left(\hat{\phi}_{i}^{(k+1)},\right. & \left.\hat{\omega}_{d_{i}}^{(k+1)}\right) \\
= & \underset{\hat{\phi}_{i}, \hat{\omega}_{d_{i}}}{\arg \max }\left[\mathbf{X}^{\mathrm{H}}\left(\hat{\omega}_{d_{i}}^{(k)}\right) \mathbf{a}\left(\hat{\phi}_{d_{i}}^{(k)}\right), \mathbf{X}^{\mathrm{H}}\left(\hat{\omega}_{d_{i}}^{(k)}\right) \mathbf{a}\left(\hat{\phi}_{s_{i}}^{(k)}\right)\right] B^{-1} \\
& \times\left[\mathbf{X}^{\mathrm{H}}\left(\hat{\omega}_{d_{i}}^{(k)}\right) \mathbf{a}\left(\hat{\phi}_{d_{i}}^{(k)}\right), \mathbf{X}^{\mathrm{H}}\left(\hat{\omega}_{d_{i}}^{(k)}\right) \mathbf{a}\left(\hat{\phi}_{s_{i}}^{(k)}\right)\right]^{\mathrm{H}} .
\end{aligned}
$$

It can be found that using the iterative process described above, the high-dimensional search of multiple targets can be simplified into parameter estimation of only one target. Therefore, the computational load can be reduced greatly compared with (6). With the echo of the $i$ th target $\hat{x}_{i}^{(k+1)}$ known, its covariance matrix can be obtained as follows

$$
\mathbf{R}_{\hat{\phi}_{i}^{(k+1)}}=\hat{x}_{i}^{(k+1)}\left(\hat{x}_{i}^{(k+1)}\right)^{\mathrm{H}} .
$$

Since $\mathbf{R}_{\hat{\phi}_{i}^{(k+1)}}$ only contains the information of the $i$ th target, the noise subspace $\mathbf{P}_{\hat{N}_{i}^{(k+1)}}$ can be constructed after the eigendecomposition of $\mathbf{R}_{\hat{\phi}_{i}^{(k+1)}}$, and then it will be easy to use the GMUSIC algorithm to estimate the direction of the $i$ th target. As has been assumed above that every target echo contains both the direct wave and the multipath component. The two-dimensional spatial spectrum of $i$ th target can be expressed as [17]

$$
\begin{aligned}
& S_{\rho}\left(\hat{\phi}_{d_{i}}^{(k+1)}, \hat{\phi}_{s_{i}}^{(k+1)}\right) \\
& \quad=\left[\min \frac{\rho^{\mathrm{H}} \mathbf{A}^{\mathrm{H}}\left(\hat{\phi}_{d_{i}}^{(k)}, \hat{\phi}_{s_{i}}^{(k)}\right) \mathbf{P}_{\hat{N}_{i}^{(k+1)}} \mathbf{A}\left(\hat{\phi}_{d_{i}}^{(k)}, \hat{\phi}_{s_{i}}^{(k)}\right) \rho}{\rho^{\mathrm{H}} \mathbf{A}^{\mathrm{H}}\left(\hat{\phi}_{d_{i}}^{(k)}, \hat{\phi}_{s_{i}}^{(k)}\right) \mathbf{A}\left(\hat{\phi}_{d_{i}}^{(k)}, \hat{\phi}_{s_{i}}^{(k)}\right) \rho}\right]^{-1} .
\end{aligned}
$$

Equation (12) can be computed as the minimum root of the following quadratic equation

$$
\begin{aligned}
& \operatorname{det}\left\{\mathbf{A}^{\mathrm{H}}\left(\hat{\phi}_{d_{i}}^{(k)}, \hat{\phi}_{s_{i}}^{(k)}\right) \mathbf{P}_{N i} \mathbf{A}\left(\hat{\phi}_{d_{i}}^{(k)}, \hat{\phi}_{s_{i}}^{(k)}\right)\right. \\
& \left.\quad-\lambda \mathbf{A}^{\mathrm{H}}\left(\hat{\phi}_{d_{i}}^{(k)}, \hat{\phi}_{s_{i}}^{(k)}\right) \mathbf{A}\left(\hat{\phi}_{d_{i}}^{(k)}, \hat{\phi}_{s_{i}}^{(k)}\right)\right\}=0 \\
& \left|\hat{\phi}_{d_{i}}^{(k+1)}-\hat{\phi}_{d_{i}}^{(k)}\right|<\text { tolerance. }
\end{aligned}
$$

From (13), we can get the elevations $\phi_{d_{i}}$ and $\phi_{s_{i}}$ for each target. The iteration should be continued until the tolerance is satisfied. When the horizontal distance from target to antenna is given, we can obtain the height of the target.

To illustrate the proposed TS-GMUSIC method more clearly, we take the two-target case as an example. In this case, the echoes of target $P$ and target $Q$ are received in the beam of the groundwork radar; the echoes of the two targets with direct waves and multipath waves are mixed together in the receiver. The transmitting signal is assumed as

$$
S_{e}(t)=s(t) e^{j 2 \pi f t} q(t)
$$


where $s(t)$ denotes the transmitted signal with different modulation, $f$ is the central carrier-frequency, $q(t)$ denotes the temporal rectangular window of length $T_{p}$, and $T_{p}$ is the pulsewidth. Normally, the large time-bandwidth product signal (linear FM (LFM) or step frequency) can be used as the transmitted signal.

Target $P$ and target $Q$ are characterized by a delay $\tau$ and a Doppler shift $\omega_{d}$, and their coordinates are $\left(R_{P 0}, \theta_{P}, \phi_{P}, \omega_{d_{P}}\right)$ and $\left(R_{Q 0}, \theta_{Q}, \phi_{Q}, \omega_{d_{Q}}\right)$, respectively. It is known that the time delay $\tau$ is related with the target parameters, including the initial range $R_{0}$, azimuth $\theta$, elevation $\phi$, and Doppler $\omega_{d}$. Then the received echo is the mixture of the transmitted signal modulated by different target information. The received target echo of the $l$ th antenna corresponding to the $i$ th target can be expressed as

$$
x_{l}(t)=a_{l}\left(t-\tau_{\left(R_{i}, \theta_{i}, \phi_{i}, \omega_{d_{i}}\right)}\right) S_{T}\left(t-\tau_{\left(R_{i 0}, \theta_{i}, \phi_{i}, \omega_{d_{i}}\right)}\right)+u_{l}(t)
$$

where $a_{l}\left(t-\tau_{\left(R_{i}, \theta_{i}, \phi_{i}, \omega_{d_{i}}\right)}\right)$ is the envelope variation containing the backward scattering amplitude of the $l$ th antenna. This item can be omitted in the narrowband system, while in the wideband system, it can be compensated. After the frequency mixture process, the received echo can be expanded as

$$
\begin{aligned}
x_{l}(t)= & s\left(t-\tau_{\left(R_{i 0}, \theta_{i}, \phi_{i}, \omega_{d_{i}}\right)}\right) \exp \left\{-j 2 \pi f \tau_{\left(R_{i 0}, \theta_{i}, \phi_{i}, \omega_{d_{i}}\right)}\right\} \\
& \times q\left(t-\tau_{\left(R_{i 0}, \theta_{i}, \phi_{i}, \omega_{d_{i}}\right)}\right)+u_{l}(t) \\
= & s\left(t-\tau_{i 0}\right) \cdot \Phi_{i \theta} \cdot \Phi_{i \phi} \cdot \Phi_{\omega_{d_{i}}}+u_{l}(t)
\end{aligned}
$$

where $\tau_{i 0}=2 R_{i 0} / C$ is the time delay of the initial range, and $C$ is the light speed. The target range $R_{i 0}$ can be obtained after the matched-filtering or correlation process. In this process, we multiply the above echo by a referenced signal,

$$
x_{l}\left(R, \theta, \phi, \omega_{d}\right)=x_{l}(t) \cdot s_{\mathrm{ref}}^{*}(t) .
$$

Normally, the transmitted signal is taken as the referenced signal.

With the targets in the different range cell distinguished, we now consider the targets in the same range cell as depicted in Fig. 2. Since the targets $P$ and $Q$ are in the same range cell, the echo can be expressed as

$$
\begin{aligned}
x_{l}\left(\theta, \phi, \omega_{d}\right) & =x_{l P}\left(\theta_{P}, \phi_{P}, \omega_{d_{P}}\right)+x_{l Q}\left(\theta_{Q}, \phi_{Q}, \omega_{d_{Q}}\right) \\
& =\alpha_{R_{0}} \cdot \Phi_{\theta} \cdot \Phi_{\phi} \cdot \Phi_{\omega_{d}}+u_{l}(t)
\end{aligned}
$$

where $\alpha_{R_{0}}$ is the pulse compression coefficient, and $x_{l}\left(\theta, \phi, \omega_{d}\right)$ contains two target echoes with different elevations and azimuths. The initial estimation of the direction parameters can be carried out by using the traditional beamforming at the beginning of the tracing. Since the targets $P$ and $Q$ are in different azimuths, their echoes can be separated respectively from the received echoes by iterative projection. That is to say, the influence of target $Q$ is eliminated from the received echoes to obtain more precise estimation of target $P$. In the $k$ th iteration, the echo of target $Q$ can be constructed with the previously estimated parameters

$$
\begin{aligned}
x_{Q}^{(k-1)} & \left(\hat{\theta}_{Q}^{(k-1)}, \hat{\phi}_{Q}^{(k-1)}, \hat{w}_{d_{Q}}^{(k-1)}\right) \\
& =\alpha_{R_{0}} \cdot \exp \left\{-j 2 \pi f \tau_{\left(\hat{\theta}_{Q}^{(k-1)}, \hat{\phi}_{Q}^{(k-1)}, \hat{w}_{d_{Q}}^{(k-1)}\right)}\right\} \\
& =\alpha_{R_{0}} \cdot \Phi_{\theta_{Q}} \cdot \Phi_{\phi_{Q}} \cdot \Phi_{w_{d_{Q}}} .
\end{aligned}
$$

Then the constructed echo of target $P$ can be picked out by projecting (18) onto the noise subspace

$$
\begin{gathered}
N_{\left(\hat{\theta}_{Q}^{(k-1)}, \hat{\phi}_{Q}^{(k-1)}, \hat{w}_{d_{Q}}^{(k-1)}\right)} \text { of } x_{Q}^{(k-1)}, \\
x_{P}^{(k)}=N_{\left(\hat{\theta}_{Q}^{(k-1)}, \hat{\phi}_{Q}^{(k-1)}, \hat{w}_{d_{Q}}^{(k-1)}\right)} \cdot x_{l}\left(\theta, \phi, w_{d}\right) \\
N_{\left(\hat{\theta}_{Q}^{(k-1)}, \hat{\phi}_{Q}^{(k-1)}, \hat{w}_{d_{Q}}^{(k-1)}\right)}=I-\hat{P}_{\left(\hat{\theta}_{Q}^{(k-1)}, \hat{\phi}_{Q}^{(k-1)}, \hat{w}_{d_{Q}}^{(k-1)}\right)} \\
\hat{P}_{\left(\hat{\theta}_{Q}^{(k-1)}, \hat{\phi}_{Q}^{(k-1)}, \hat{w}_{d_{Q}}^{(k-1)}\right)}=x_{Q}^{(k-1)}\left[\left(x_{Q}^{(k-1)}\right)^{\mathrm{H}} x_{Q}^{(k-1)}\right]\left(x_{Q}^{(k-1)}\right)^{\mathrm{H}}
\end{gathered}
$$

where $\hat{P}_{\left(\hat{\theta}_{Q}^{(k-1)}, \hat{\phi}_{Q}^{(k-1)}, \hat{w}_{d_{Q}}^{(k-1)}\right)}$ is the signal subspace of target $Q$ in the $(k-1)$ th iteration, which is formed by the constructed echo $x_{Q}^{(k-1)} \cdot x_{Q}^{(k-1)}$ can be viewed as the direction vector of target $Q$ in the $(k-1)$ th iteration. After the subspace projection, the echo of target $P$ is left in the residual echo. Since only one target information remains in the residual echo, the problem of target direction estimation in (6) can be changed into that of one target parameter estimation, as shown in (10). Next we compute the covariance matrix according to (11), and then make the spectral estimation using (12); with the parameters of target $P$ obtained, we can construct $x_{P}^{(k)}\left(\hat{\theta}_{P}^{(k)}, \hat{\phi}_{P}^{(k)}, \hat{w}_{d_{P}}^{(k)}\right)$ by (19). The parameters of target $Q$ can be achieved by the same way described above. The iterative procedure will be continued until the tolerance of (13b) is satisfied.

Finally, considering the range and elevation of the target as well as the curvature radius of the Earth, we can get the altitude of each target as

$$
\hat{H}_{i}^{(k)}=R_{i 0} \sin \left(\hat{\phi}_{d_{i}}^{(k)}\right)+H_{a}+\frac{R_{i 0}^{2}}{2 R_{e}}, \quad i=P, Q
$$

where $R_{e}$ is the radius of the Earth, $H_{a}$ is the height of the antenna, and $R_{i 0}$ is the initial range of the $i$ th target.

\section{An Improved Algorithm}

In this subsection, in order to simplify the two-dimensional direction search, we consider the multipath geometry characteristic of the low-elevation target in the far field scenario. Here we suppose the flying height of the target is relatively low over the 
sea surface as depicted in Fig. 1. In this circumstance, we can find that the elevations $\phi_{d}$ and $\phi_{s}$ are related by

$$
\frac{R_{d} \cos \left(\phi_{d}\right)}{\sin \left(90-\phi_{s}\right)}=\frac{H_{r}+H_{t}}{\sin \left(\phi_{s}\right)}
$$

With (22), a two-dimensional parameter estimation problem can be efficiently changed into a one-dimensional one. In addition, by incorporating the multipath geometry as a priori information, the proposed TS-GMUSIC algorithm shows better performance as illustrated in the simulation results given in the next section.

\section{SIMULATION RESULT AND THE PROCESS OF REAL DATA}

In this section, we give three simulations to validate the proposed TS-GMUSIC algorithm. The first simulation shows the effect of SNR on the proposed method. The second simulation compares the proposed method with the typical superresolution algorithms to illustrate its advantages. And the third simulation applies the proposed method to real-data processing.

In the first simulation, we employ a uniform linear array with 16 antennas which are equally spaced half a wavelength apart. The radar is placed at the height of $100 \mathrm{~m}$ above the ground. The central frequency is $240 \mathrm{MHz}$. In the far field, two low-altitude Doppler signals with the same SNR are impinging on the array. We denote these two targets as target A and target B. For the two targets, their horizontal distances to the center of the antennas are both $100 \mathrm{~km}$, their SNR are both $20 \mathrm{~dB}$, and their azimuths are $90^{\circ}$ and $95^{\circ}$, respectively. The altitude and Doppler frequency of target A are, respectively, $2200 \mathrm{~m}$ and $60 \mathrm{~Hz}$. For target B, its altitude is $2000 \mathrm{~m}$, and the Doppler frequency is $88 \mathrm{~Hz}$. The magnitude of the

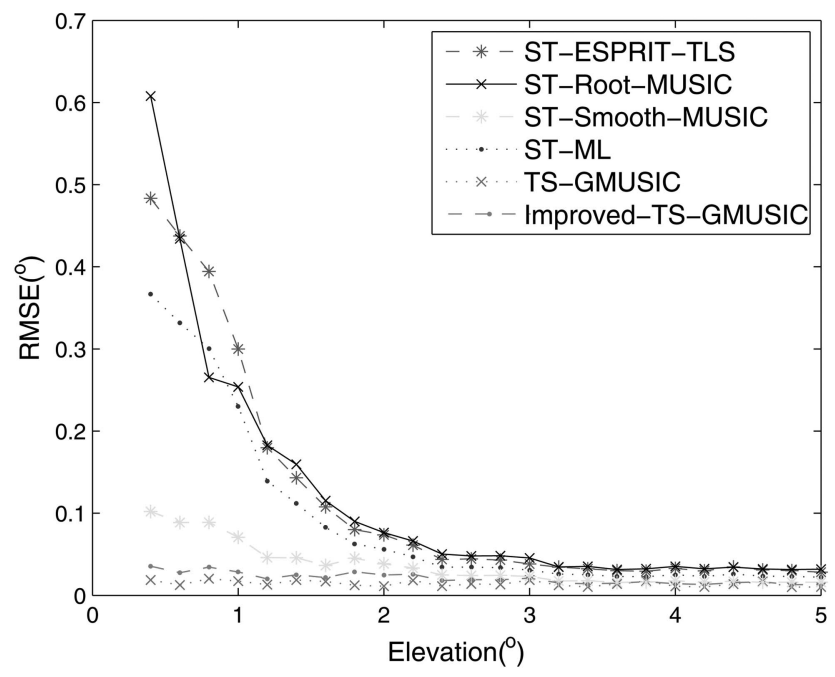

(a)

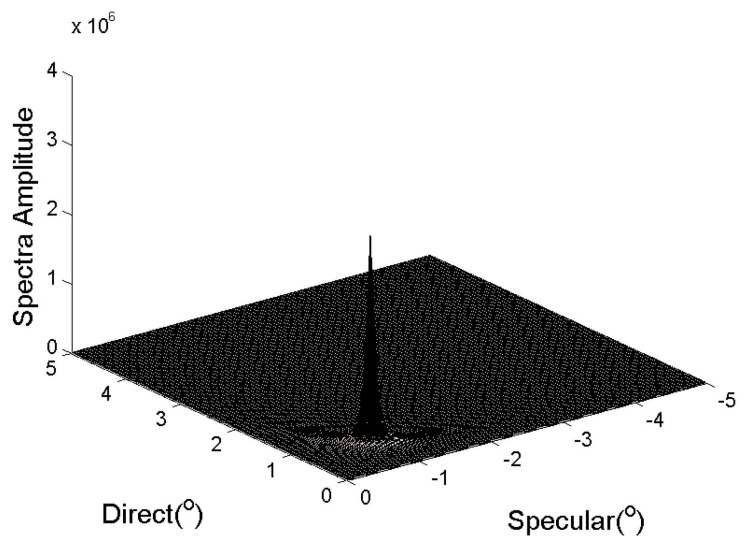

Fig. 3. Spatial spectrum estimation of target A.

surface reflection coefficient is 0.95 . Fig. 3 shows the spatial spectrum of target A by using the proposed TS-GMUSIC algorithm. The measurement results of the altitude of the two targets are $2216 \mathrm{~m}$ and $1998 \mathrm{~m}$, respectively.

We can see from Fig. 3 that, by using the proposed method, the simulation result is very close to the true altitudes of the targets. Under the high SNR, the projection can obtain only one target echo without the interference of the other target. Thus the resulting measurement is much more precise. While when the SNR is below $13 \mathrm{~dB}$, the received echo after the projection will contain the information of other target, it degrades the precision of the measurement, even with the presence of pseudo peak.

In the second simulation, we compare the proposed TS-GMUSIC, the improved method, and typical subspace-based superresolution algorithms, such as ESPRIT, root-MUSIC [18] and smooth-MUSIC. Fig. 4(a) shows the root-mean-square error (RMSE) of estimation with the elevation varying from $0.4^{\circ}$ to $5^{\circ}$. To test the validity of the proposed

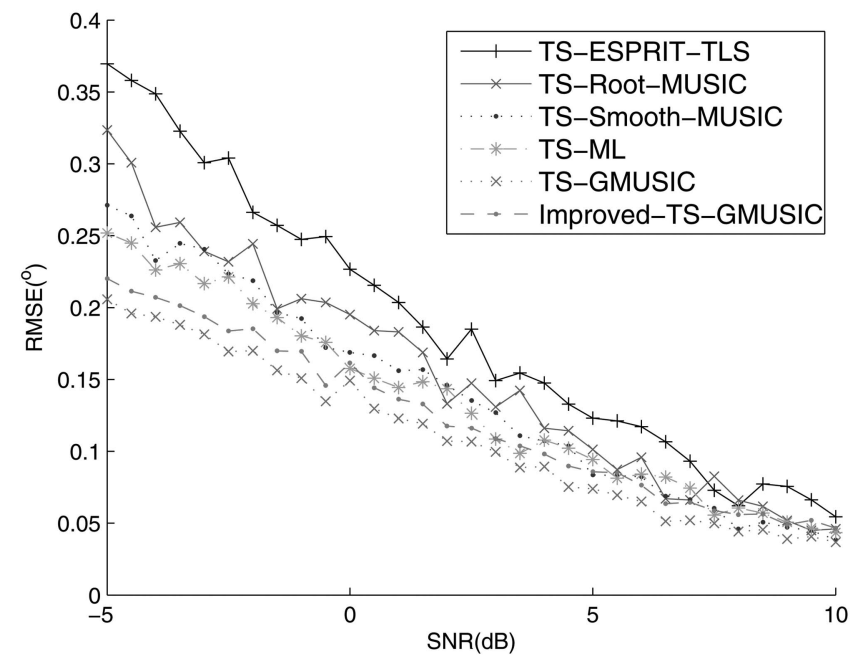

(b)

Fig. 4. Comparison of Monte-Carlo experiment. (a) RMSE versus different elevation. (b) RMSE versus different SNR. 
method in different SNR, we set the target altitude and the initial range to be, respectively, $2000 \mathrm{~m}$ and $100 \mathrm{~km}$. The Monte-Carlo experiment with 100 trials is given in Fig. 4(b) with the SNR varying from $-5 \mathrm{~dB}$ to $10 \mathrm{~dB}$. The SNR depicted here is the value before pulse compression.

It could be found that from Fig. 4, when the elevation of the target is approaching zero, subspace-based algorithms cause great error. Especially compared with the TS-ML method, our proposed method eliminates the affect of other targets, and thus the result is much more precise. Furthermore, the proposed method achieves higher estimation precision over other methods even in the low SNR case.

To further demonstrate the performance of the proposed method, we compared it with the TS-ML algorithm in terms of the computational load of each iteration. In the proposed TS-GMUSIC method, for each iteration, we need to calculate the matrix inversion and the projection only once. While in the TS-ML algorithm, for each iteration, the matrix inversion and the projection need to be calculated at least twice. The computational load of the matrix inversion is $N^{3}$, where $N$ is the dimension of the matrix. So the proposed method can reduce the computational complexity greatly. To validate the above analysis, under the same design parameters, a test of convergence speed is shown in Fig. 5.

It can be found that since the TS-ML algorithm needs multi-dimensional search, its error estimation of the angle at the beginning is better than the proposed TS-GMUSIC. With the increase of the projection precision, the proposed method shows more precise angle estimation, and the corresponding time consumption is greatly reduced compared with the TS-ML algorithm. Consideration of the prior

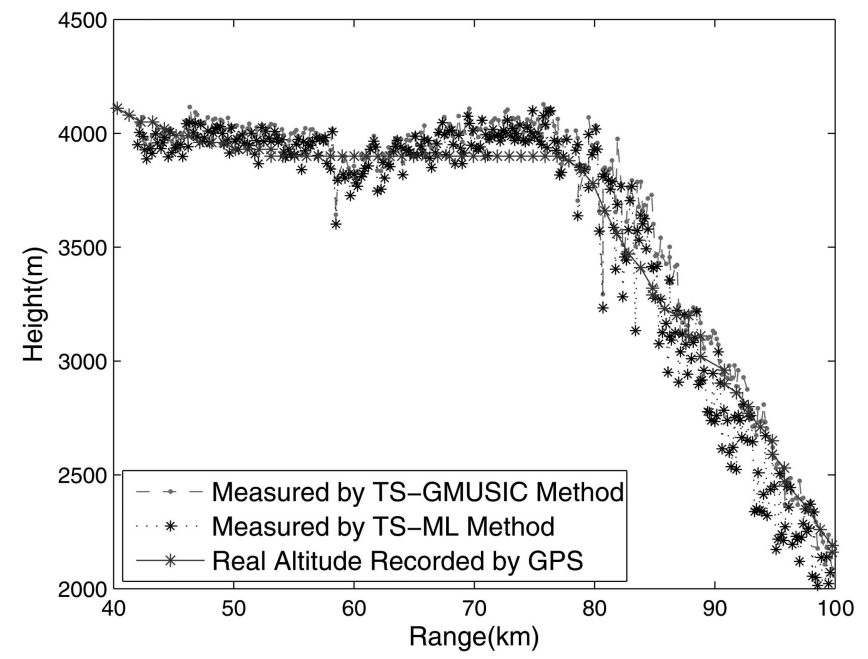

(a)

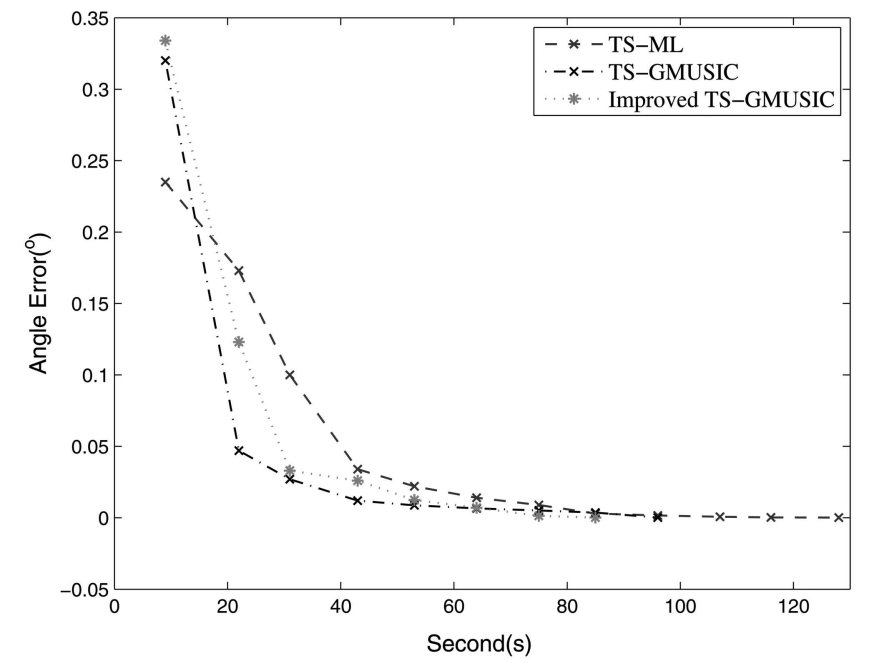

Fig. 5. Comparison of converge speed.

knowledge of the multipath geometry reduces the computational load to a certain degree.

In the third simulation, we apply the proposed method to the real data of a set of aeroplanes recoded from the VHF radar. The VHF radar is set beside a lake $40 \mathrm{~m}$ higher than the horizontal level. The antenna is made up of a planar array which has two line subarrays, each of which has a uniform linear array with 16 sensors. The distance between every two adjacent elements is $1.1 \mathrm{~m}$, and the sampling frequency is $1000 \mathrm{~Hz}$. First, we use (20) to separate the targets by projection, and then we track two of the targets and measure their altitudes. Fig. 6(a) shows the altitude measurement comparison of a falling plane, and Fig. 6(b) shows that of a takeoff plane. From the tracking, we can find that the landing velocity of the target in Fig. 6(a) is from $508 \mathrm{~km} / \mathrm{h}$ to $443 \mathrm{~km} / \mathrm{h}$, and the target in Fig. 6(b) is from $397 \mathrm{~km} / \mathrm{h}$ to $596 \mathrm{~km} / \mathrm{h}$, respectively.

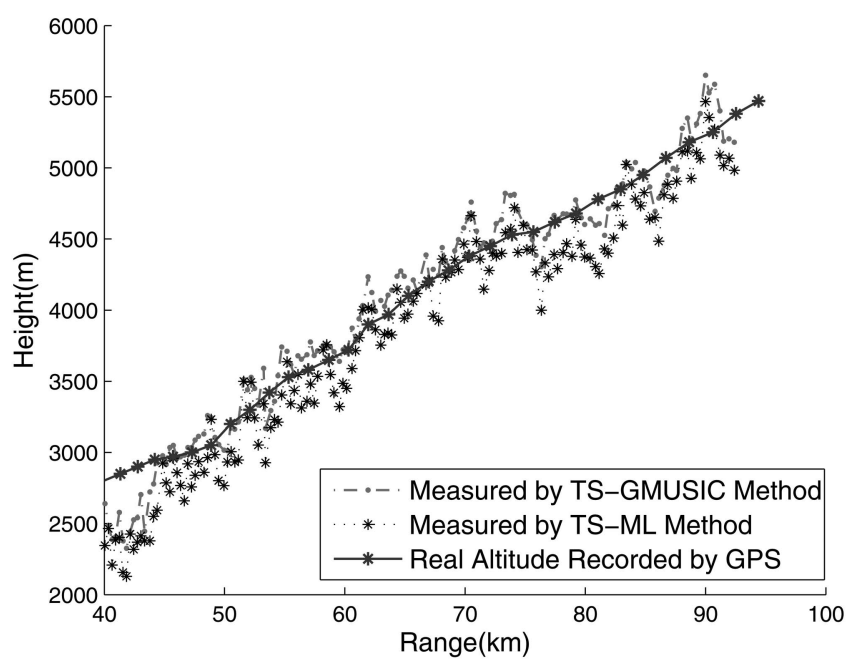

(b)

Fig. 6. Estimation result of real data of VHF radar. (a) Altitude measurement comparison of landing plane. (b) Altitude measurement comparison of takeoff plane. 
We can see from Fig. 6 that the result of the proposed method approaches the real altitude recorded by GPS. For the TS-ML algorithm, since its beam direction is affected by the energy of the specular target, the target direction is lower than the real target elevation, and the corresponding target altitude is lower than the GPS measurement.

\section{SUMMARY AND CONCLUSION}

In this paper, we propose a TS-GMUSIC algorithm for low-elevation target direction search. The proposed algorithm constructs the received echo of each target based on the iterative projection theory. The direction and altitude can be obtained by processing the covariance matrix of a single target echo. The simulation results and the real data experiment prove that our proposed method has better estimation precision and less computational load.

\section{REFERENCES}

[1] Barton. D. K. Low-elevation radar tracking. Proceedings of the IEEE, 62, 6 (June 1974), 687-704.

[2] Modurasinghe, D. Direction finding in a multipath environment. Electronics Letters, 27 (Jan. 1991), 61-62.

[3] Guldogan. M. B. and Arikan, O. Performance analysis of cross ambiguity function-Direction finding technique in multipath environments.

In Proceedings of the 16th IEEE Signal Processing, Communication Applications Conference, SIU 2009, Apr. 2008, 1-4.

[4] Wang, X. and Musicki, D.

Low elevation sea-surface target tracking using IPDA type filters.

IEEE Transactions on Aerospace and Electronic Systems, 43, 2 (Apr. 2007), 759-774.

[5] Capon, J.

Nonlinear methods of spectral analysis. In S. Haykin (Ed.), Maximum-Likelihood Spectral Estimation, New York: Springer-Verlag, 1979, ch. 5.

[6] Hussain, M. G. M.

Principles of space-time array processing for ultrawide-band impulse radar and radio communications. IEEE Transactions on Vehicular Technology, 51, 3 (May 2002), 393-403.

[7] Friedlander, B. Direction finding using a spatial smoothing with interpolated arrays.

IEEE Transactions on Aerospace and Electronic Systems, 28, 2 (Apr. 1992), 574-587.
[8] Hyberg, P., Jansson, M., and Ottersten. B. Array interpolation and bias reduction. IEEE Transactions on Signal Processing, 52, 10 (Oct. 2004), 2711-2720.

[9] Dogan, M. C. and Mendel, J. M. Applications of cumulants to array processing-Part I: Aperture extension and array calibration. IEEE Transactions on Signal Processing, 43, 5 (May 1995), 1200-1216.

[10] Dogan, M. C. and Mendel, J. M. Applications of cumulants to array processing-Part IV: Direction finding in coherent signals case. IEEE Transactions on Signal Processing, 45, 9 (Sept. 1997), 2265-2276.

[11] Schmidt, R. O.

Multiple emitter location and signal parameter estimation. IEEE Transactions on Antennas and Propogation, AP-34, 3 (May 1986), 276-280.

[12] Wong, K. T. and Zoltowski, M. D.

Uni-vector-sensor ESPRIT for multi-source azimuth-elevation angle estimation.

IEEE Transactions on Antennas and Propogation, 45, 10 (Oct. 1997), 1467-1474.

[13] Shan, T. J., Wax, M., Kailath, T.

On spatial smoothing for direction-of-arrival estimation of coherent signals.

IEEE Transactions on Acoustics, Speech and Signal

Processing, ASSP-33, 8 (Aug. 1985), 806-811.

[14] Pillai, S. U. and Kwon, B. H.

Forward/backward spatial smoothing techniques for coherent signal identification.

IEEE Transactions on Acoustics, Speech and Signal Processing, 37, 1 (Jan. 1989), 8-15.

[15] Ziskind, I. and Wax, M.

Maximum likelihood localization at multiple sources by alternating projection.

IEEE Transactions on Acoustics, Speech and Signal

Processing, 36, 10 (Oct. 1988), 1553-1560.

[16] Yongkang, J. and Zheng, B.

DOA estimation methods and its performance for signals with temporal-spatial 2-dimension model.

Acta Electronica Sinica, 25, 9 (1997), 69-73.

[17] Zoltowski, M.

Vector space approach to direction finding in a coherent multipath environment.

IEEE Transactions on Antennas and Propagation, AP-34, 9 (Sept. 1986), 1069-1079.

[18] Zoltowski, M. D., Kautz, G. M., and Silverstein, S. D. Beamspace root-MUSIC.

IEEE Transactions on Signal Processing, 41, 1 (Jan. 1993), 344-364. 

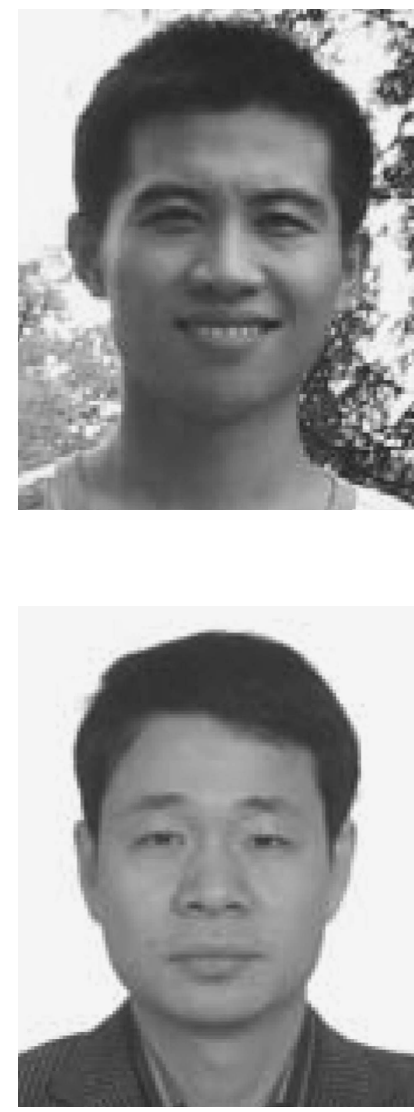

Guanghui Zhao received the B.S., M.S., and Ph.D. degrees in electronical and information engineering from Xidian University, China, in 2004, 2007, and 2008.

$\mathrm{He}$ is now working as a teacher at Xidian University. His research activities are target altitude measurement in VHF radar, array spectrum estimation theory, array processing with applications to MIMO radar, and application of the compressive sensing theory in radar system.

Guangming Shi received the B.S., M.S., and Ph.D. degrees from Xidian University, China, in 1985, 1988, and 2002, respectively.

He joined the Department of Electronic Engineering, Xidian University, in 1988. Since 2003, he has been a professor in the School of Electronic Engineering and in 2004 became the Head of National Instruction Base of Electrician \& Electronic (NIBEE). Presently, he is the Deputy Director of the School of Electronic Engineering and the academic leader in the subject of electronic circuit and system. His current research interests are in compressive sensing theory, image processing (denoising, debluring, and coding), high resolution imaging, UWB signal sampling, and embedded systems. 